\title{
Dissociating explicit and implicit measures of sensed hand position in tool use: Effect of relative frequency of judging different objects
}

\author{
Miya K. Rand ${ }^{1} \cdot$ Herbert Heuer ${ }^{1}$ \\ Published online: 26 October 2017 \\ (C) The Psychonomic Society, Inc. 2017
}

\begin{abstract}
In a cursor-control task, the sensed positions of cursor and hand are biased toward each other. We previously found different characteristics of implicit and explicit measures of the bias of sensed hand position toward the position of the cursor, suggesting the existence of distinct neural representations. Here we further explored differences between the two types of measure by varying the proportions of trials with explicit hand-position $(\mathrm{H})$ and cursor-position $(\mathrm{C})$ judgments (C20:H80, C50:H50, and C80:H20). In each trial, participants made a reaching movement to a remembered target, with the visual feedback being rotated randomly, and subsequently they judged the hand or the cursor position. Both the explicitly and implicitly measured biases of sensed hand position were stronger with a low proportion (C80:H20) than with a high proportion (C20:H80) of hand-position judgments, suggesting that both measures place more weight on the sensory modality relevant for the more frequent judgment. With balanced proportions of such judgments (C50:H50), the explicitly assessed biases were similar to those observed with a high proportion of cursor-position judgments (C80:H20), whereas the implicitly assessed biases were similar to those observed with a high proportion of hand-position judgments (C20:H80). Because strong weights of cursor-position or hand-position information may be difficult to increase further but are easy to reduce, the findings suggest that the implicit measure of the bias of sensed hand position places a relatively stronger weight on proprioceptive hand-position information, which is increased no further by a high proportion of hand-position judgments.
\end{abstract}

Miya K. Rand

rand@ifado.de

1 Leibniz Research Centre for Working Environment and Human Factors (IfADo), Ardeystraße 67, 44139 Dortmund, Germany
Conversely, the explicit measure places a relatively stronger weight on visual cursor-position information.

Keywords Reaching $\cdot$ Tool use $\cdot$ Sensory integration · Implicit measure $\cdot$ Explicit measure

Perception relies on multiple sources of information, and multisensory integration of redundant estimates of a certain property of an object serves to increase the precision of perception. Multisensory integration is generally optimal or almost so in that the weights of the integrated estimates are specified in proportion to their relative reliabilities. This goes along with minimal variance (or maximal precision) of the combined perceptual estimate (e.g., Cheng, Shettleworth, Huttenlocher, \& Rieser, 2007; Ernst \& Banks, 2002; Ernst \& Bülthoff, 2004). The benefits of multisensory integration are obvious as long as the various estimates refer to the same property of the same object - for example, the position of the hand (van Beers, Sittig, \& Denier van der Gon, 1999). However, a weaker form of multisensory integration, called sensory coupling (e.g., Bresciani, Dammeier, \& Ernst, 2006), has also been observed in tool use, in particular in cursor-control tasks, where proprioception refers to the position of the hand and vision to the position of a cursor. Although the hand and cursor are clearly different objects, estimates of their respective positions become biased toward each other (Debats, Ernst, \& Heuer, 2017a; Kirsch, Herbort, Ullrich, \& Kunde, 2017; Kirsch, Pfister, \& Kunde, 2016; Ladwig, Sutter, \& Müsseler, 2012, 2013; Rand \& Heuer, 2013, 2016, 2017). These biases depend not only on the sensory input - that is, on the proprioceptive and visual information on hand and cursor position, respectively-but also on how the biases are assessed (cf. Debats, Ernst, \& Heuer, 2017b). Here we explore differences between two types of assessment that we designate 
as explicit and implicit measures of the bias of sensed hand position.

The explicit measure of the bias of sensed hand position is based on psychophysical judgments. After a reaching movement of constant amplitude and variable direction and the return to the starting position, participants move their hand along a circular path to its remembered position at the end of reaching. These judgments are psychophysical judgments of the perceived and remembered positions of the hand that participants are consciously aware of. The deviation of these judgments from the physical position of the hand serves as an explicit measure of the bias of sensed hand position toward the position of the cursor. The implicit measure of the bias of sensed hand position, in contrast, is based on the movements of the participants without them being aware of judging the position of their hand. As is detailed in the Method section, this measure is the angle between the reaching movement and the return movement back to the starting position. Its rationale is based on the observation that discrepancies between the sensed and physical locations of the hand give rise to systematic errors in subsequent movements (Bock \& Eckmiller, 1986; Heuer \& Sangals, 1998; Heuer \& Sülzenbrück, 2012; Holmes, Crozier, \& Spence, 2004; Holmes \& Spence, 2005; Rossetti, Desmurget, \& Prablanc, 1995).

In previous studies, we observed a number of differences between the implicit and explicit measures (Rand \& Heuer, 2013, 2016, 2017). The explicit measure of the bias of sensed hand position is substantially stronger and more variable than the implicit measure. Individual explicitly and implicitly assessed biases are uncorrelated. Only the explicitly measured bias, but not the implicitly measured bias, becomes stronger at older adult age (Rand \& Heuer, 2013), weaker with increased relative reliability of proprioceptive input (Rand \& Heuer, 2016), and interindividually less variable after visuomotor adaptation (Rand \& Heuer, 2017). These differences and dissociations are consistent with the notion of two different neural representations of hand position (e.g., Dijkerman \& de Haan, 2007; de Vignemont, 2010; Head \& Holmes, 1911; Paillard, 1991), similar to cognitive (perceptual, explicit) and motor (action-oriented, implicit) representations of visual stimuli (e.g., Bridgeman, Lewis, Heit, \& Nagle, 1979; Milner \& Goodale, 1995, 2008).

In the present study, we continued our search for differences between explicit and implicit assessments of the bias of sensed hand position toward the position of the cursor. In particular, we compared the effects of different proportions of trials in which participants have to judge explicitly either the position of the hand or the position of the cursor. Our hypothesis that this manipulation might affect explicitly and implicitly assessed biases differently was based on the following considerations. First, different proportions of trials in which cursor and hand positions have to be judged should result in a stronger or a weaker weight of the hand position in the coupled estimate. A stronger or a weaker weight of the hand position in the coupled estimate, which is conceptualized as a weighted average, implies a weaker or a stronger bias, respectively. Second, with equal proportions of trials with handposition and cursor-position judgments, hand position might have a relatively stronger weight for implicitly assessed biases than for explicitly assessed biases. A stronger weight should be more easily reduced than increased, and a weaker weight should be more easily increased than reduced. We elaborate these considerations in more detail.

Among the classic findings on reaction times is the relative-frequency effect: In a binary choice task, the reaction time to the more frequent of two signals is faster than the reaction time to the less frequent signal (e.g., Fitts, Peterson, $\&$ Wolpe, 1963). The more frequent signal is also more frequently expected, with faster reaction times for correctly than for incorrectly expected signals (Hinrichs, 1970), and expectations of the more frequent signal are more frequently correct. The higher relative frequency of one of two types of trials goes along with a higher number of repetitions, and repetitions typically facilitate performance (cf. Cheng, Luis, \& Tremblay, 2008; Khan, Elliott, Coull, Chua, \& Lyons, 2002; Kirby, 1980; Song \& Nakayama, 2007; Zelaznik, Hawkins, \& Kisselburgh, 1983). Notably, as compared with alternations between two types of trials, repetitions of one type facilitate the selective processing of individual stimulus features. This is evident from the costs of switching between tasks as compared with task repetitions, in which tasks are defined by the selective processing of one of at least two different stimulus features (see Kiesel et al., 2010; Monsell, 2003, for reviews). Findings such as these suggest that relative frequency can serve to modulate the selectivity of information processing.

In the present experiment, we varied the proportions of trials with explicit judgments of cursor $(\mathrm{C})$ and hand $(\mathrm{H})$ position in ratios of $\mathrm{C} 80: \mathrm{H} 20$ and $\mathrm{C} 20: \mathrm{H} 80$. We expected that with these different proportions, either the visual information on cursor position (with $\mathrm{C} 80: \mathrm{H} 20$ ) or the proprioceptive information on hand position (with $\mathrm{C} 20: \mathrm{H} 80$ ) would become prioritized so that its weight in sensory coupling would be increased. We had two reasons for this expectation. First, the more frequently given judgments could result in more reliable estimates of either cursor position or hand position, and a higher reliability would be associated with a stronger weight (cf. Debats et al., 2017b). Second, the weights in sensory coupling are also likely to depend on factors other than relative reliabilities (cf. Sober \& Sabes, 2005). Thus, they might also be affected directly by the experimental conditions, not mediated by the different reliabilities of the perceptual estimates. In terms of observed biases rather than weights in sensory coupling, the bias of sensed hand position toward the cursor position should be stronger with a higher proportion of cursor-position judgments than with a higher proportion of 
hand-position judgments. This should be observed for explicitly and/or implicitly assessed biases. For the bias of sensed cursor position toward the hand position, the effects of the different relative frequencies should be the opposite. This can be observed for the explicitly assessed bias of sensed cursor positions only because we are not aware of a suitable implicit procedure comparable to our implicit assessment of the bias of sensed hand position.

The second consideration that gave rise to our hypothesis that implicit and explicit measures of the bias of sensed hand position toward the position of the cursor might be affected differently by different proportions of trials with explicit judgments of cursor and hand positions was the following: With a C50:H50 proportion, hand position might have a relatively stronger weight for the implicitly than for the explicitly assessed bias. In principle, different weighting for psychophysical judgments and motor responses would be possible (Knill, 2005). A stronger weight for hand-position information could be the reason for the consistently smaller size of the implicitly assessed bias of sensed hand position, which would go along with a smaller intra-individual variability (Rand \& Heuer, 2013, 2016, 2017). If indeed hand position has a stronger weight for the implicit than for the explicit assessment, it might be difficult to increase the weight any further. This would be consistent with our previous finding that additional proprioceptive input served to strengthen the hand-position weight for the explicit assessment, but not for the implicit assessment (Rand \& Heuer, 2016).

Taking into account all the considerations, we tentatively expected the following effects from three conditions with different proportions of trials of explicit cursor-position and hand-position judgments-C80:H20, C50:H50, and C20:H80. (1) For C20:H80, the hand-position weight in sensory coupling should be stronger than the same weight in $\mathrm{C} 80: \mathrm{H} 20$. Therefore, the bias of the sensed hand position toward the position of the cursor should be weaker. (2) This difference between the two conditions might not be the same for implicit and explicit assessments. (3) For explicit assessments of cursor position, the difference between the C20:H80 and C80:H20 conditions should be the opposite: The cursorposition weight in sensory coupling should be weaker in condition $\mathrm{C} 20$ :H80, and thus, the bias of the sensed cursor position toward the position of the hand should be stronger. (4) Regarding the $\mathrm{C} 50: \mathrm{H} 50$ condition, there should be a stronger weight of hand position for implicit than for explicit assessments. Thus, there might be a smaller increase of its weight (and decrease of its bias) with a higher proportion of hand-position judgments in condition C20:H80 for implicit than for explicit assessments. With the smaller proportion of hand-position judgments in condition $\mathrm{C} 80: \mathrm{H} 20$, in contrast, there might be a greater decrease of the handposition weight (and increase of the bias) for implicit than for explicit assessments.

\section{Materials and method}

\section{Participants}

Twenty-eight healthy right-handed young adults (mean $[S D]$ age: 22.6 [2.8] years; 15 males and 13 females) signed informed consent prior to participating in the study. The study was conducted in accordance with the Declaration of Helsinki and with the approval of the ethics committee of the Leibniz Research Centre for Working Environment and Human Factors.

\section{Apparatus and procedure}

The experimental setting and procedures were similar to those in our previous studies (Rand \& Heuer, 2013, 2016). In brief, seated participants held a stylus with their right hand and made three-stroke movements on a digitizer (Wacom Intuos $4 \mathrm{XL}$, $133-\mathrm{Hz}$ sampling rate) while looking at a monitor. The monitor was covered by a large black circular screen with a semicircular window ( $32 \mathrm{~cm}$ in diameter) in its center. A first target (T1, $1.4 \mathrm{~cm}$ in diameter) was located in that center, and the start position (SP, $1.2 \mathrm{~cm}$ in diameter) was located $3 \mathrm{~cm}$ below $\mathrm{T} 1$. A second target (T2, $1 \mathrm{~cm}$ in diameter) was presented at pseudo random locations, ranging from $-60^{\circ}$ to $+60^{\circ}$ relative to the central location, on an invisible circle with a radius of $15 \mathrm{~cm}$ around T1. Corresponding to this circle, the workspace on the digitizer was bordered by a semi-circular plastic ring, the "stopper ring," with a radius of $15 \mathrm{~cm}$ around T1. An opaque board placed above the participants' arm blocked their direct view of the hand movements.

At the beginning of each trial, participants were guided to the SP by arrows shown on the monitor. One second after the stylus was in the SP, T2 appeared for $1 \mathrm{~s}$ (Fig. 1a, 1st panel). Subsequently, T1 appeared. After a delay of $0.5 \mathrm{~s}$, an auditory go signal was delivered. The participants then made three stroke movements, from the SP to T1 (1st stroke), to T2 (2nd stroke), and back to T1 (3rd stroke), at a comfortable speed. When the 1st stroke reached T1, this target disappeared. Then the participants made the 2 nd stroke to the remembered T2 (Fig. 1a, 3rd panel) until the movement was stopped by the stopper ring. Afterward, they made a return movement (3rd stroke) back to the remembered T1 location (Fig. 1a, 4th panel).

The participants made the 1st and 2nd strokes with concurrent visual feedback provided by a cursor on the monitor, but the 3 rd strokes without visual feedback. During the 2nd strokes, however, the direction of cursor motion was rotated relative to the direction of hand movement by an angle randomly chosen out of six (clockwise [CW] direction: $-25^{\circ}$, $15^{\circ},-5^{\circ}$; counterclockwise $[\mathrm{CCW}]$ direction: $5^{\circ}, 15^{\circ}, 25^{\circ}$ ). Thus, the participants had to adjust their movements so that 
a Cursor position judgment

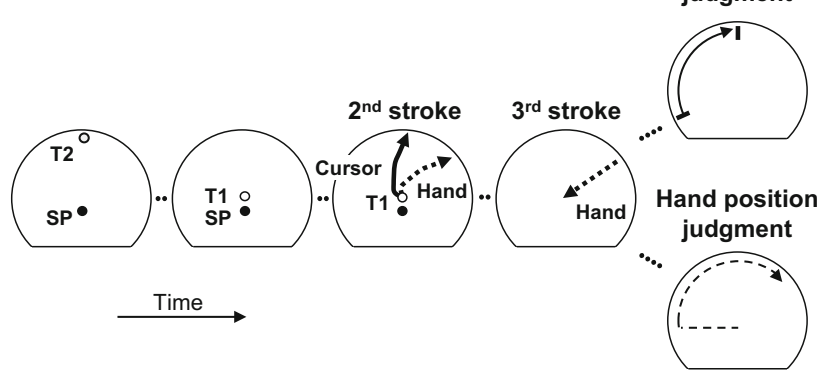

b

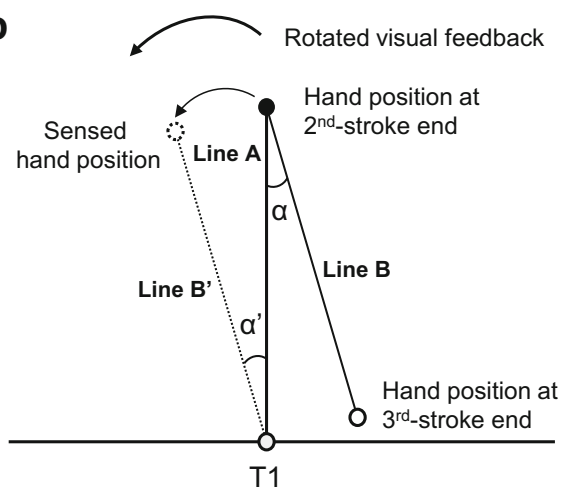

Fig. 1 Behavioral task of a three-stroke movement and judgments of hand and cursor positions. (a) SP, T1, and T2 refer to a starting position, a first target, and a second target, respectively. The visual feedback of the 2nd stroke is rotated and displayed simultaneously with the hand movements. After the 2 nd stroke, the participants make a return movement without the visual feedback as the 3rd stroke, and subsequently make an explicit judgment regarding the hand or cursor position. Arrows with a solid line refer to motions of the visual feedback (3rd panel) and a direction maker (5th panel, top). Arrows with a dashed line refer to hand movements (not the visual feedback). (b) Implicit measure of hand position. The directional deviation $\alpha^{\prime}$ of the sensed hand position (dotted outline circle) from the physical one (black circle) at the end of the 2 nd stroke is estimated from the directional deviation $\alpha$ of the hand position at the end of the 3rd stroke (solid outline circle) from its remembered target (T1, gray circle).

the cursor on the monitor would move toward the remembered T2 location (Fig. 1a, 3rd panel).

One second after completion of the 3rd stroke (Fig. 1a, 4th panel), the participants were asked to judge either the hand or the cursor position at the end of the 2 nd stroke. To indicate the type of judgment to be performed, either the word "Hand" (for a judgment of hand position) or "Cursor" (for a judgment of cursor position) appeared briefly in the center of the monitor, together with an arrow pointing to either the right (for a judgment using a CCW motion) or the left (for a judgment using a $\mathrm{CW}$ motion). Thence, the specified judgment followed.

For the judgment of cursor position (explicit cursor judgment; Fig. 1a, 5th panel, top), a short line moved either counterclockwise or clockwise along the edge of the display window at a constant speed. The participant instructed the examiner to stop and finely adjust (back and/or forth) the line to the position that matched the remembered position of the cursor at the end of the 2nd stroke. For the judgment of hand position (explicit hand judgment; Fig. 1a, 5th panel, bottom), the participant moved the pen from the right (or left) lower corner of the stopper ring counterclockwise (or clockwise) along the ring and stopped where he or she judged the hand position to match the remembered position of the hand at the end of the 2nd stroke. Note that the motion of the visual marker and the movement of the hand during the judgments differed from the cursor motion and hand movement, respectively, during the 2nd stroke. Therefore, only positions on the circular path could be matched, but not the movement directions and/ or amplitudes of the 2nd strokes. The type of explicit judgment (hand or cursor position) was randomized across trials, but with different relative frequencies according to experimental condition. The direction of line or hand movement during the judgment (clockwise or counterclockwise) was randomized across trials with the constraint of equal frequencies.

The three experimental conditions differed in the relative frequencies of trials with explicit judgments of hand and cursor position. The first condition had proportions of $80 \%$ cursor-judgment trials (144 trials) and 20\% hand-judgment trials (36 trials), and is referred to as C80:H2O. The second condition (C50:H50) had proportions of $50 \%$ cursorjudgment trials ( 90 trials) and 50\% hand-judgment trials ( 90 trials), and the third condition (C20:H80) had 20\% cursorjudgment trials (36 trials) and $80 \%$ hand-judgment trials (144 trials). The experiment was conducted over three days (one condition per day). The order of conditions was approximately counterbalanced across participants.

Before the $\mathrm{C} 80: \mathrm{H} 20$ (or $\mathrm{C} 20: \mathrm{H} 80$ ) condition, the participants were informed that most of the trials would require cursor (or hand) judgments. They were also told that although once in a while different judgments would be required, the best strategy would be to pay attention to the cursor (or hand) position. Before the $\mathrm{C} 50: \mathrm{H} 50$ condition, the participants were informed that cursor and hand judgments would occur equally often, and they were instructed to pay attention to both the cursor and hand positions in each trial.

Before data recording on the first day of the experiment, eight familiarization trials without the judgment were performed that included the procedure with/without the visualfeedback rotation, as well as four warm-up trials that included the entire procedure (two trials each for hand and cursor judgments). The same four warm-up trials were also performed on the second and third days of the experiment. There were six sets of trials (each of which included one warm-up trial and 30 experimental trials) for each condition. A short break was inserted after each set. A total of 540 experimental trials (180 trials per day) were recorded and analyzed for each participant. 


\section{Data analysis}

The data analyses were similar to those of our previous studies (Rand \& Heuer, 2013, 2016). In brief, positions were measured in a polar coordinate system with the origin in T1, and only the angles were analyzed because the distance from the origin was constant for all judged and physical positions of the hand and cursor at the end of the 2 nd stroke. In each trial, the angular deviation of the judged hand or cursor position from the actual hand or cursor position at the end of the 2nd stroke was determined (the CCW direction had a positive sign) and served as the explicit measure of the bias in that trial. Individual means and standard deviations of the angular deviations were computed for the judged directions of cursor and hand for each condition (C80:H20, C50:H50, or C20:H80) and each visual-feedback rotation. The overall influence of the rotated visual feedback on the angular deviations of the judged from the actual hand or cursor positions was assessed by the slope of the linear regression of the angular deviation (dependent variable) on the visual-feedback rotation (independent variable), which was computed for each participant, experimental condition, and judged position (cursor or hand). These slope parameters specify the strength of sensory coupling in terms of the proportional biases of the judgments of hand and cursor positions in degree per degree of the visualfeedback rotation.

As an implicit measure of the bias of sensed hand position toward the position of the cursor in each trial, we computed the angular deviation of the direction of the 3rd stroke from the direction of the 2nd stroke - that is, $\alpha^{\prime}=\alpha$ in Fig. 1b. This measure exploits the existence of error propagation in successive aiming movements, in particular the propagation of errors that originate from visually induced deviations between the physical and sensed positions of the hand. In our case, the visually induced deviations of the sensed position of the hand from the actual position at the end of the 2nd stroke (angle $\alpha^{\prime}$ in Fig. 1b) resulted from the rotated visual feedback, and these deviations were estimated from the angular error of the return movement (angle $\alpha$ in Fig. 1b). When the sensed position deviated in the $\mathrm{CCW}$ or $\mathrm{CW}$ direction from the actual position of the hand, the angular deviation $\left(\alpha^{\prime}\right)$ had a positive or negative sign, respectively. Individual means and standard deviations of the angular deviations were computed for each experimental condition and each visual-feedback rotation across trials with explicit hand-position judgments. Trials with explicit cursor-position judgments were neglected for the implicit measure of the bias of sensed hand direction, so that the explicit and implicit measures would be based on the same set of trials. The angular deviations $\alpha^{\prime}$ were subjected to the same linear regressions as the angular deviations of the explicit judgments of hand position from the actual position, to obtain a measure of the implicitly assessed bias as a proportion of the visual-feedback rotation.
The data were screened for outliers both among trials and among participants. On the basis of the linear regressions applied separately for each type of bias assessment (explicit cursor, explicit hand, implicit hand), each condition (C80:H20; $\mathrm{C} 50: \mathrm{H} 50, \mathrm{C} 20: \mathrm{H} 80$ ), and each participant, trials with angular deviations outside the range of predicted deviations \pm 3 standard deviations of the residuals were eliminated as outliers among the trials. In total, $0.48 \%$ of experimental trials were removed from all analyses. Subsequently, the bias parameters for each type of assessment and each condition were screened for outliers among participants. The means and standard deviations across all participants were calculated for the three types of measurement and the three conditions, and bias parameters outside the range of mean \pm 3 standard deviations were defined as outliers. These computations were repeated until no further outliers were found. As the result, two participants were identified as having outliers for the explicitly assessed bias of cursor direction and were excluded from all analyses.

The individual regression coefficients of the explicit and implicit bias measures of hand direction were subjected to a 3 (Proportion: C80:H20, C50:H50, C20:H80) $\times 2$ (Type of Measure: hand-explicit, hand-implicit) repeated measures analysis of variance (ANOVA). Our main interest was in the interaction, which would indicate different effects of the different proportions of cursor-judgment and hand-judgment trials on the explicit and implicit measures of the bias of sensed hand position toward the position of the cursor. For these interaction tests, we split the three-level factor proportion into two orthogonal contrasts, with coefficients $-1,0,1$ (linear trend) and 1, $-2,1$ (quadratic trend) for the conditions $\mathrm{C} 80: \mathrm{H} 20$, C50:H50, and C20:H80, respectively. The interaction of type of measure with the first of these contrasts tests whether the difference between $\mathrm{C} 80: \mathrm{H} 20$ and $\mathrm{C} 20: \mathrm{H} 80$ is different between the two types of measure. The interaction with the second contrast tests whether the deviation from equal differences between the three proportions (nonlinearity) is different for the explicit and implicit measures. (More specifically, for conditions C80:H20, C50:H50, and C20:H80, we assigned coefficients $-1,0,1$ for the explicit measure and 1 , $0,-1$ for the implicit one, respectively, to test the interaction of type of measure with the linear trend of the proportion, and coefficients $1,-2,1$ for the explicit measure and, $-1,2,-1$ for the implicit one, respectively, to test the interaction of type of measure with the quadratic trend of the proportion).

We ran additional analyses for the explicitly assessed bias of sensed cursor position together with the explicitly assessed bias of sensed hand position and for the individual standard deviations (i.e., intra-individual variation) of the biases. The explicit biases of sensed cursor and hand position were analyzed in the same way as the explicit and implicit biases of sensed hand position. The individual standard deviations of the implicit and explicit measures of the bias of sensed hand 
position were subjected to a 3 (Proportion: $\mathrm{C} 80: \mathrm{H} 20$, C50:H50, C20:H80) $\times 2$ (Type of Measure: hand-explicit, hand-implicit $) \times 6$ (Visual-Feedback Rotation) repeated measures ANOVA, and the individual standard deviations of the explicit measures of the bias of sensed cursor position and sensed hand position were subjected to the same type of ANOVA. Mauchly's test was used to test for violations of sphericity. In case of violations, Greenhouse-Geisser epsilon is reported, and statistical significance was evaluated using the corrected degrees of freedom.

\section{Results}

The main purpose of the present study was to test whether explicit and implicit measures of the bias of sensed hand position toward the position of the cursor are affected differently by variation of the proportions of trials with explicit cursorposition and hand-position judgments. The respective findings are presented first. Next, we report the intra-individual variability of implicit and explicit measures of the bias of sensed hand position and finally the analyses of the explicit measures of the bias of sensed cursor and hand positions.

\section{Explicit and implicit measures of the bias of sensed hand position}

The mean angular deviations of the judged hand position from the physical position showed steep positive slopes as a function of the visual-feedback rotation for all three different proportions of cursor-position and hand-position judgments (Fig. 2a, hand-explicit), indicating a strong explicitly assessed bias toward the position of the cursor. As for the implicit measure of the bias of sensed hand position, the mean angular deviations of the return movements also had positive slopes as a function of the visual-feedback rotation for all different proportions of explicit cursor-position and hand-position judgments (Fig. 2a, hand implicit), again indicating a bias toward the cursor position. However, these slopes were less steep than those observed for the explicit measure. A 3 (Proportion) $\times 2$ (Type of Measure) ANOVA of the individually estimated biases (slopes of the linear regressions) revealed the main effect of type of measure to be significant $[F(1,25)=44.48$, $p<.001]$.

The means of the individually estimated biases (Fig. 2b) show more clearly than Fig. $2 \mathrm{a}$ the variations across the three proportions $\mathrm{C} 80: \mathrm{H} 20$, C50:H50, and $\mathrm{C} 20: \mathrm{H} 80$ for both the explicit and implicit measures of the bias of sensed hand position. For both measures (Fig. 2b, hand-explicit and handimplicit), the bias was stronger in condition $\mathrm{C} 80: \mathrm{H} 20$ - that is, with a strong weight on the cursor position in the coupled estimate - than in condition $\mathrm{C} 20: \mathrm{H} 80$ - that is, with a strong weight on the hand position in the coupled estimate. The
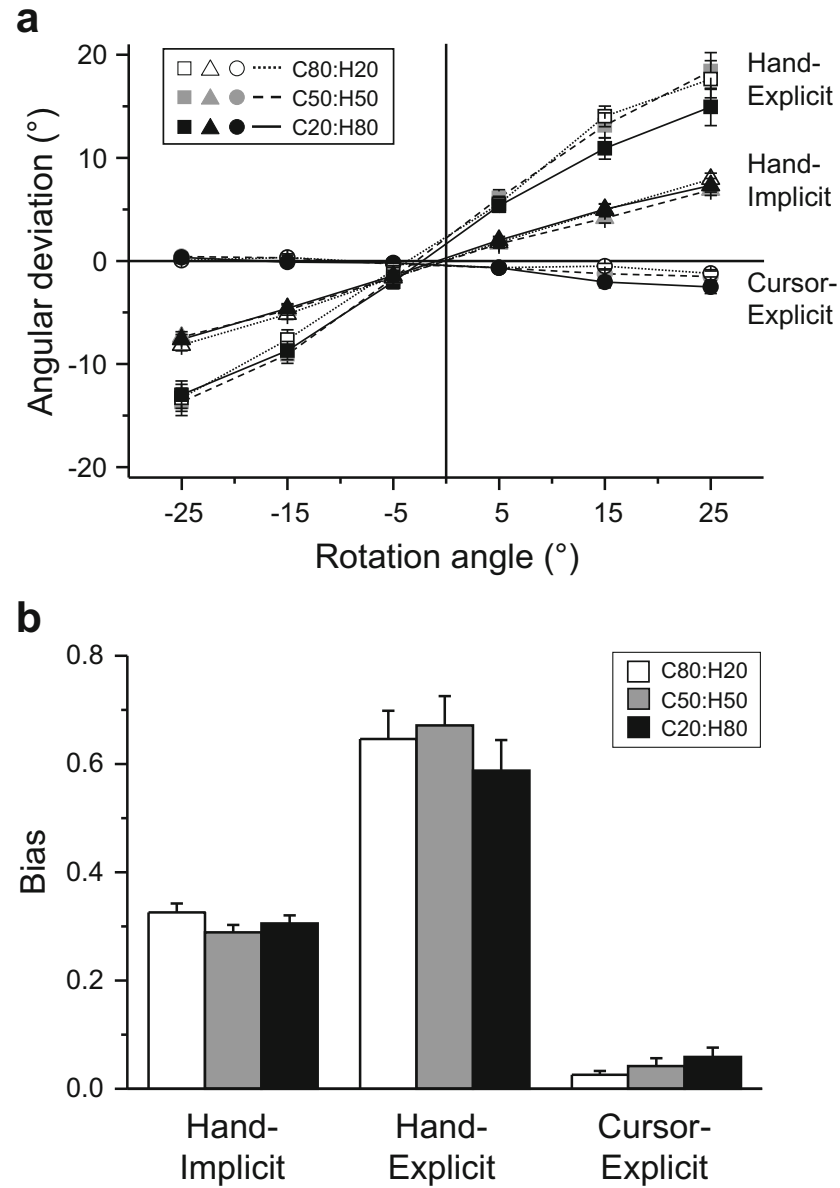

Fig. 2 (a) Mean explicit and implicit measures as a function of the rotation of the visual feedback. The mean values across all participants are plotted for the explicit measure of hand position (squares), the implicit measure of hand position (triangles), and the explicit measure of cursor position (circles). (b) Mean explicitly measured proportional biases of hand position (hand-explicit), implicitly measured proportional biases of hand position (hand-implicit), and explicitly measured proportional biases of cursor position (cursor-explicit) are plotted for the C80:H20 (white columns), C50:H50 (gray columns), and C20:H80 (black columns) conditions. The error bars represent the standard errors.

ANOVA revealed that the main effect of proportion was marginally significant $[F(2,50)=3.10, p=.054]$. The linear trend of the proportion effect (based on the contrast coefficients 1, 0 , -1 being assigned for both types of measure to conditions $\mathrm{C} 80: \mathrm{H} 20$, C50:H50, and C20:H80, respectively) was significant $[F(1,25)=8.65, p=.007]$, indicating that the $\mathrm{C} 80: \mathrm{H} 20$ and C20:H80 conditions differed significantly when the two types of measure were pooled. Conversely, the quadratic trend of the proportion effect (based on the contrast coefficients 1,2, 1 being assigned for both types of measure to conditions $\mathrm{C} 80: \mathrm{H} 20, \mathrm{C} 50: \mathrm{H} 50$, and $\mathrm{C} 20: \mathrm{H} 80$, respectively) was not significant $[F(1,25)=0.64, p=.430]$, indicating no nonlinearity of the $\mathrm{C} 50: \mathrm{H} 50$ condition relative to the other two conditions when both types of measure were pooled.

As for the interaction between the type of measure and proportion, the linear trend of proportion (contrast coefficients 
1, 0, - 1 for conditions C80:H20, C50:H50, and C20:H80; see the details in the Method section) was not significantly different between the explicit and implicit measures $[F(1,25)=2.74$, $p=.111]$. This indicates that the differences between conditions C80:H20 and C20:H80 were similar for the two types of measure. In contrast, the conspicuous difference between the two measures was the bias in the condition C50:H50. The bias in this condition was close to the bias in condition C80:H20 (strong weight of cursor position) and different from the bias in condition C20:H80 (strong weight of hand position) for the explicit measure. For the implicit measure, in contrast, the bias in condition $\mathrm{C} 50: \mathrm{H} 50$ was close to the bias in condition $\mathrm{C} 20$ :H80 (strong weight of hand position) and different from the bias in condition C80:H20 (strong weight of cursor position). This dissociation was confirmed by a significant difference $[F(1,25)=7.42, p=.012]$ of the quadratic trends of proportions between the two types of measure (contrast coefficients $1,-2,1$ for conditions C80:H20, C50:H50, and C20:H80; see the details in the Method section).

\section{Intra-individual variability of biases of sensed hand position}

In Fig. 3a, the mean intra-individual standard deviations of explicitly and implicitly assessed biases of sensed hand position are plotted as a function of the rotation of visual feedback. The individual standard deviations of the implicit measure (Fig. 3a, hand-implicit) were substantially smaller than those of the explicit measure (hand-explicit), which was confirmed by a significant main effect of type of measure $[F(1,25)=206.27$, $p<.001]$ in a 3 (Proportion) $\times 2$ (Type of Measure) $\times 6$ (Visual-Feedback Rotation) ANOVA. We found no systematic differences between the three proportions C80:H20, C50:H50, and $\mathrm{C} 20: \mathrm{H} 80[F(2,50)=0.34, p=.711]$. The main effect of visual-feedback rotation was significant $[F(5,125)=9.59$, $\varepsilon=.61, p<.001]$, and so was the interaction between type of measure and visual-feedback rotation $[F(5,125)=3.04$, $p=.013]$. The increase of intra-individual variability at the largest visuomotor rotations was somewhat stronger for the explicit than for the implicit measure (Fig. 3a). No other significant effects were found.

\section{Explicit measures of the biases of sensed cursor and hand positions}

The mean angular deviations of the judged cursor position from the corresponding physical position showed slightly negative slopes as a function of the visual-feedback rotation (Fig. 2a, cursor-explicit), indicating a weak bias of the judgments of cursor position toward the position of the hand. The individually estimated biases (slopes of the linear regressions) were multiplied by -1 to make them comparable with the biases of sensed hand position, and their means are plotted
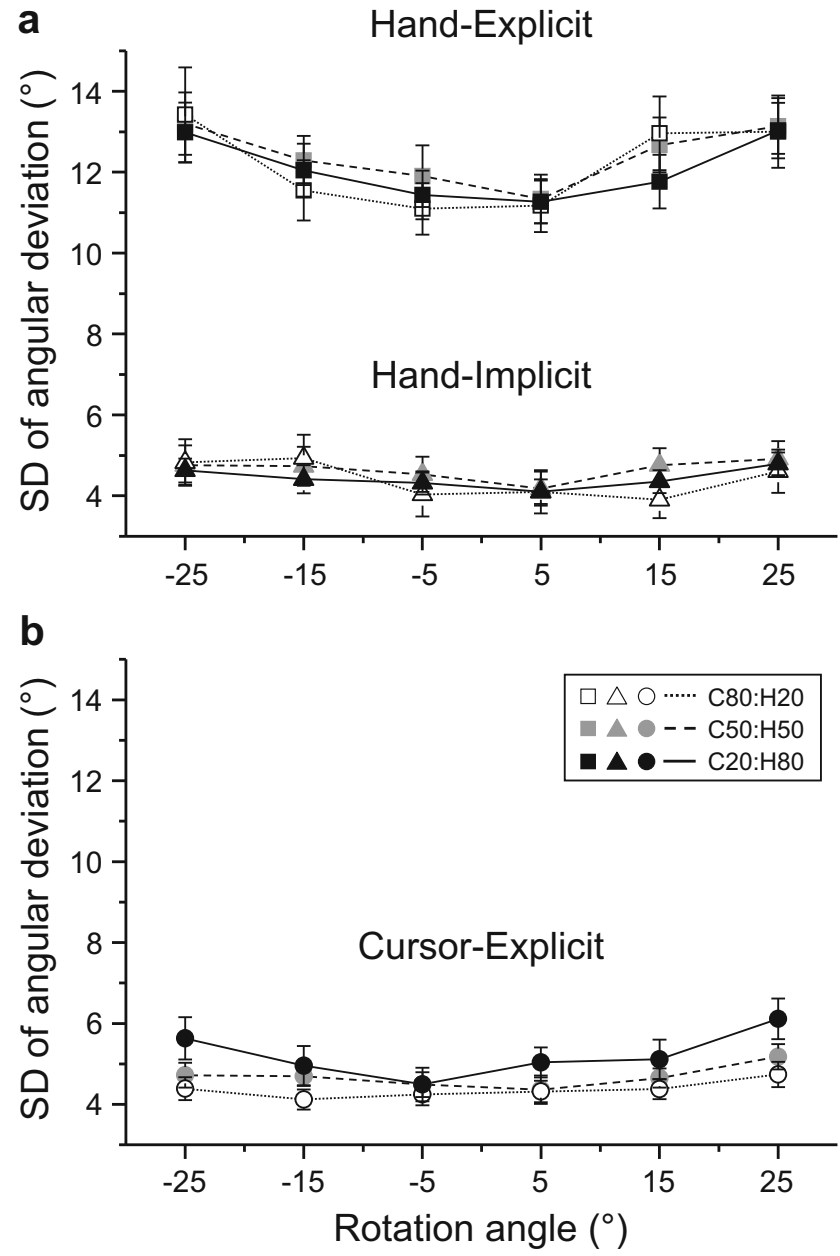

Fig. 3 Mean standard deviations $(S D)$ of the explicit and implicit measures as a function of rotation of the visual feedback. (a) The $S D$ s are plotted for explicit measures of hand position (squares) and implicit measures of hand position (triangles). (b) The $S D$ s are plotted for explicit measures of cursor position (circles). The error bars represent the standard errors.

in Fig. $2 b$ (cursor-explicit). The 3 (Proportion) $\times 2$ (Type of Measure) ANOVA showed that the obviously stronger bias of hand-position judgments than of cursor-position judgments gave rise to a significant main effect of type of measure $[F(1,25)=111.41, p<.001]$, whereas the main effect of proportion was not significant $[F(2,50)=2.29, p=.112]$.

As for the interaction between the proportion and type of measure, the bias of sensed cursor position (cursor-explicit) was weaker in condition $\mathrm{C} 80: \mathrm{H} 20$ than in condition $\mathrm{C} 20: \mathrm{H} 80$, which contrasted with the results for the bias of sensed hand position (Fig. 2b, hand-explicit). Thus, there was a trade-off between the explicitly assessed biases of sensed hand position and sensed cursor position, the former being stronger in condition $\mathrm{C} 80: \mathrm{H} 20$ and the latter being weaker. The linear trend of proportion (contrast coefficients $1,0,-1$ for conditions $\mathrm{C} 80: \mathrm{H} 20, \mathrm{C} 50: \mathrm{H} 50$, and $\mathrm{C} 20: \mathrm{H} 80$; see the details in the Method section) was significantly different between the explicit measures of the biases of sensed cursor and hand 
position $[F(1,25)=10.69, p=.003]$, whereas the quadratic trend (contrast coefficients 1, $-2,1$ for conditions C80:H20, C50:H50, and C20:H80; see the details in the Method section) was not significantly different $[F(1,25)=2.33, p=.140]$.

The nonsignificant main effect of proportion above (i.e., the variation of the means of the hand and cursor biases across the three proportions of hand and cursor judgments) implies that the variation of the sum of the biases was also nonsignificant. The sum of the hand and cursor biases is an estimate of the strength of sensory coupling, independent of the asymmetry of the biases (cf. Debats et al., 2017b). The means (and standard errors) of the coupling strength were $.67 \pm .051, .71 \pm .056$, and $.65 \pm .050$ for the conditions $\mathrm{C} 80: \mathrm{H} 20, \mathrm{C} 50: \mathrm{H} 50$, and $\mathrm{C} 20: \mathrm{H} 80$, respectively.

\section{Intra-individual variability of explicitly assessed biases}

The mean intra-individual standard deviations of the explicit measure of the bias of sensed cursor position were generally small (Fig. 3b), considerably smaller than those of the explicit measure of the bias of sensed hand direction (Fig. 3a, handexplicit). The 3 (Proportion) $\times 2$ (Type of Explicit Measure) $\times$ 6 (Visual-Feedback Rotation) ANOVA showed that the difference between the two explicit measures was significant $[F(1$, $25)=224.60, p<.001]$. In addition, we observed a significant main effect of visual-feedback rotation $[F(5,125)=9.45, \varepsilon=$ $.64, p<.001]$ and an interaction between type of explicit measure and visual-feedback rotation $[F(5,125)=2.65, \varepsilon=$ $.71, p=.044]$. No other significant effects were found, including no systematic differences between the three conditions C80:H20, C50:H50, and C20:H80. Nevertheless, it is noteworthy that the smaller bias of cursor judgments in condition C80:H20 than in condition $\mathrm{C} 20: \mathrm{H} 80$ was associated with a smaller variability in condition C80:H20 than in C20:H80 (4.36 versus $5.22 \mathrm{deg}$ ), whereas the stronger bias of hand judgments in condition C80:H20 was associated with a slightly larger variability than in condition C20:H80 (12.20 versus $12.09 \mathrm{deg}$ ). The interaction of type of measure with the linear trend of proportion (contrast coefficients 1,0 , 1 for conditions C80:H20, C50:H50, and C20:H80; see the details in the Method section) fell just short of statistical significance $[F(1,25)=3.12, p=.089]$.

\section{Discussion}

The main results of the present study are: (1) both the explicitly and implicitly assessed biases of sensed hand position toward the position of the cursor were stronger with a low proportion of hand-position judgments (C80:H20) than with a high proportion (C20:H80); (2) the explicitly assessed bias of sensed cursor position toward the position of the hand showed the opposite pattern-namely, weaker bias with a low proportion of hand-position judgments $(\mathrm{C} 80: \mathrm{H} 20)$ than with a high proportion (C20:H80); (3) with a balanced proportion (C50:H50), the explicitly assessed bias of sensed hand position was similar to the one observed with a high proportion of cursor-position judgments (C80:H20), whereas the implicitly assessed bias of sensed hand position was similar to the one observed with a high proportion of hand-position judgments (C20:H80). We discuss these findings in turn.

\section{Commonalities of implicit and explicit measures of the bias of sensed hand position}

Both the explicitly and implicitly assessed biases of sensed hand position decreased when the proportion of handposition judgments increased from $20 \%$ to $80 \%$ of the trials, and this decrease was similar in magnitude for both types of measure. A decrease of the bias of sensed hand position can indicate a reduction of the strength of sensory coupling or a change of its asymmetry (cf. Debats et al., 2017b). Sensory coupling implies mutual biases, which are biases of sensed hand position and of sensed cursor position in the present study. When the strength is reduced, both biases become smaller, so that their sum becomes smaller. When the asymmetry is changed, the one bias is reduced whereas the other one is increased, so that their sum remains unchanged. The present results of the explicitly assessed bias of sensed hand position showed clear evidence that its reduction at a higher proportion of trials with explicit hand-position judgments is due to a change of the asymmetry of sensory coupling, not to a change of its strength. At a higher proportion of trials with hand-position judgments, the explicitly assessed bias of sensed cursor position was increased, and this increase compensated the reduction of the bias of sensed hand position. The sum of both biases, that is, the measure of coupling strength, remained unchanged.

For the implicitly assessed bias of sensed hand position, we are neither aware of a corresponding implicit assessment of the sensed cursor position nor did we measure it in this study. Hence, we found no direct evidence that the change of the implicitly assessed bias of sensed hand position was also due to a change of the asymmetry of sensory coupling, rather than to a reduction of coupling strength. However, there is indirect evidence that a modulation of the asymmetry of sensory coupling by different proportions of explicit handposition and cursor-position judgments was present for the explicitly assessed bias. At the same time, there are no obvious reasons why coupling strength should be modulated only for the implicitly assessed bias - which would be different from the explicit assessment of the bias.

How do the effects of the relative proportions of trials with explicit cursor-position and hand-position judgments on the asymmetry of sensory coupling come about? Although the findings on attentional effects on sensory coupling are rather mixed (e.g., Macaluso et al., 2016; Odegaard, Wozny, \& 
Shams, 2016; Talsma, Doty, \& Woldorff, 2007; Talsma, Senkowski, Soto-Faraco, \& Woldorff, 2010), the present findings suggest that a higher proportion of hand-position judgments results in focusing attention on the relevant proprioceptive information, and a higher proportion of cursor-position judgments in focusing attention on the relevant visual information. Thus, relative frequency among two types of judgments may serve to modulate the attentional selectivity and the related information processing.

A strong determinant, though not the only one, of the weights in sensory coupling is the reliability of the individual estimates of cursor position and hand position (cf. Debats et al., 2017b). The present findings on the modulation of the asymmetry of sensory coupling induced by different proportions of cursor-position and hand-position judgments might well be mediated by the relative reliabilities of the individual estimates that result from the modulation of attentional selectivity. In particular, with a high frequency of hand-position judgments, attention might be focused on proprioceptive information, resulting in a high reliability of hand-position estimates and low reliability of cursor-position estimates. With a high frequency of cursor-position judgments, conversely, attention might be focused on visual information, resulting in a high reliability of cursor-position estimates and low reliability of hand-position estimates. Such hypothetical differences in reliability of the individual sensory estimates should to some extent be reflected in the variabilities of the coupled sensory estimates. In fact, the mean standard deviations observed with high and low proportions of explicit cursor-position and handposition judgments revealed the corresponding pattern, though statistically the interaction was nonsignificant.

\section{Differences between implicit and explicit measures of the bias of sensed hand position}

The explicitly and implicitly assessed biases of sensed hand position in the condition with equal proportions of explicit hand-position and cursor-position judgments (C50:H50) had different locations in the range of biases spanned by the conditions with an imbalance of cursor-position and handposition judgments (C80:H20 and C20:H80). More specifically, the implicit measure of the bias in condition C50:H50 was similar to that in condition $\mathrm{C} 20: \mathrm{H} 80$, which is the condition with a stronger weight on hand-position information. Conversely, the explicit measure of the bias in condition C50:H50 was similar to that in condition $\mathrm{C} 80: \mathrm{H} 20$, which is the condition with the stronger weight on cursor-position information.

These findings suggest that the implicit measure of the bias of sensed hand position places stronger weight on proprioceptive hand-position information, whereas the explicit measure places stronger weight on visual cursor-position information. We assume that a strong weight is hard to increase further, but easy to reduce, whereas a weak weight is easy to increase, but hard to reduce further. When the importance of hand-position information is further increased in condition C20:H80 as compared with condition C50:H50, the already strong weight of hand-position information for the implicit measure is not increased further, so that the implicitly assessed bias of sensed hand position is not reduced. However, the weaker weight of hand-position information for the explicit measure is increased, resulting in a reduced explicitly assessed bias of hand position. Conversely, when the importance of cursor-position information is further increased in condition C80:H20 as compared with $\mathrm{C} 50: \mathrm{H} 50$, the low weight of cursor-position information for the implicit measure is increased, so that the implicitly assessed bias of sensed hand position becomes stronger. However, the already strong weight of cursor-position information for the explicit measure is not, so that the explicitly assessed bias of sensed hand position is not increased.

The conclusion that the implicit measure of the bias of sensed hand position places relatively stronger weight on proprioceptive hand-position information and the explicit measure relatively stronger weight on visual cursor-position information is consistent with at least two earlier observations. The first one is the consistent finding that the implicitly assessed bias of sensed hand position toward the cursor position is only about half as strong as the explicitly assessed bias (Fig. 2; Rand \& Heuer, 2013, 2016, 2017). This could result from a stronger weight of hand-position information in the implicit than in the explicit measure, consistent with the smaller variability of the implicitly assessed bias than the explicitly assessed one (Fig. 3a). The second consistent finding is the effect of strengthening the proprioceptive hand-position information by providing unimodal proprioceptive input at the end of reaching (Rand \& Heuer, 2016). With a balanced proportion of hand- and cursor-position judgments (C50:H50), it resulted in a reduced explicitly assessed bias of sensed hand position, corresponding to a stronger weight of the proprioceptive hand-position information, but had no effect on the implicitly assessed bias. Thus, further strengthening of the proprioceptive input either by means of additional input or a high proportion of hand-position judgments does not affect the relative weights of the two modalities in implicit estimates of sensed hand position, but it does so in explicit estimates.

To summarize, we have previously observed a number of different properties of the implicit and explicit measures of sensed hand position, as we briefly described in the introduction (Rand \& Heuer, 2013, 2016, 2017). The present study extends the previous findings by revealing the different sensitivity of the two measures to the proportion of trials with explicit hand-position and cursor-position judgments. The differences accumulated in our series of studies support the notion of at least two distinct neural representations of hand position (e.g., de Vignemont, 2010; Dijkerman \& de Haan, 2007; Head \& Holmes, 1911; Paillard, 1991), similar to 
cognitive (perceptual, explicit) and motor action-oriented (implicit) representations of visual stimuli (e.g., Bridgeman et al., 1979; Milner \& Goodale, 1995, 2008). Of course, there may be more representations, but only two representations can be distinguished in the present study, based on two distinct measures. In spite of the differences, the explicit and implicit measures of the bias of sensed hand position also share commonalities. For example, the common variation of both types of bias measures between the lowest and highest proportions of hand-position judgments suggests that the CNS applies the reliability-based weighting principle (Debats et al., 2017b) in coupling visual and proprioceptive information for both implicit and explicit measures.

Author note This research was supported by the German Research Foundation (DFG) [grant number Ra 2183/1-3]. We thank Maleen Greine and Franziska Schywalski for their support in data collection.

\section{References}

Bock, O., \& Eckmiller, R. (1986). Goal-directed arm movements in absence of visual guidance: Evidence for amplitude rather than position control. Experimental Brain Research, 62, 451-458.

Bresciani, J.-P., Dammeier, F., \& Ernst, M. O. (2006). Vision and touch are automatically integrated for the perception of sequences of events. Journal of Vision, 6(5), 2:554-564. https://doi.org/10.1167/ 6.5.2

Bridgeman, B., Lewis, S., Heit, G., \& Nagle, M. (1979). Relation between cognitive and motor-oriented systems of visual position perception. Journal of Experimental Psychology: Human Perception and Performance, 5, 692-700. https://doi.org/10.1037/0096-1523. 5.4.692

Cheng, D. T., Luis, M., \& Tremblay, L. (2008). Randomizing visual feedback in manual aiming: Reminiscence of the previous trial condition and prior knowledge of feedback availability. Experimental Brain Research, 189, 403-410.

Cheng, K., Shettleworth, S. J., Huttenlocher, J., \& Rieser, J. J. (2007). Bayesian integration of spatial information. Psychological Bulletin, 133, 625-637. https://doi.org/10.1037/0033-2909.133.4.625

Debats, N. B., Ernst, M. O., \& Heuer, H. (2017a). Kinematic crosscorrelation induces sensory integration across separate objects. European Journal of Neuroscience. in press.

Debats, N. B., Ernst, M. O., \& Heuer, H. (2017b). Perceptual attraction in tool-use: Evidence for a reliability-based weighting mechanism. Journal of Neurophysiology, 117, 1569-1580.

de Vignemont, F. (2010). Body schema and body image—Pros and cons. Neuropsychologia, 48, 669-680.

Dijkerman, H. C., \& de Haan, E. H. F. (2007). Somatosensory processes subserving perception and action. Behavioral and Brain Sciences, 30, 189-239.

Ernst, M. O., \& Banks, M. S. (2002). Humans integrate visual and haptic information in a statistically optimal fashion. Nature, 415, 429-433. https://doi.org/10.1038/415429a

Ernst, M. O., \& Bülthoff, H. H. (2004). Merging the senses into a robust percept. Trends in Cognitive Sciences, 8, 162-169. https://doi.org/ 10.1016/j.tics.2004.02.002
Fitts, P. M., Peterson, J., \& Wolpe, G. (1963). Cognitive aspects of information processing: II. Adjustments to stimulus redundancy. Journal of Experimental Psychology, 65, 423-432.

Head, H., \& Holmes, H. G. (1911). Sensory disturbances from cerebral lesions. Brain, 34, 102-254.

Heuer, H., \& Sangals, J. (1998). Task-dependent mixtures of coordinate systems in visuomotor transformations. Experimental Brain Research, 119, 224-236.

Heuer, H., \& Sülzenbrück, S. (2012). The influence of the dynamic transformation of a sliding lever on aiming errors. Neuroscience, 207, 137-147.

Hinrichs, J. V. (1970). Probability and expectancy in two-choice reaction time. Psychonomic Science, 21, 227-228.

Holmes, N. P., Crozier, G., \& Spence, C. (2004). When mirrors lie: "Visual capture" of arm position impairs reaching performance. Cognitive, Affective, \& Behavioral Neuroscience, 4, 193-200. https://doi.org/10.3758/CABN.4.2.193

Holmes, N. P., \& Spence, C. (2005). Visual bias of unseen hand position with a mirror: Spatial and temporal factors. Experimental Brain Research, 166, 489-497.

Khan, M. A., Elliott, D., Coull, J., Chua, R., \& Lyons, J. (2002). Optimal control strategies under different feedback schedules: Kinematic evidence. Journal of Motor Behavior, 34, 45-57.

Kiesel, A., Steinhauser, M., Wendt, M., Falkenstein, M., Jost, K., Philipp, A. M., \& Koch, I. (2010). Control and interference in task switching-A review. Psychological Bulletin, 136, 849-874. https://doi.org/10.1037/a0019842

Kirby, N. (1980). Sequential effects in choice reaction time. In A. T. Welford (Ed.), Reaction times (pp. 129-172). New York, NY: Academic Press.

Kirsch, W., Herbort, O., Ullrich, B., \& Kunde, W. (2017). On the origin of body-related influences on visual perception. Journal of Experimental Psychology: Human Perception and Performance, 43, 1222-1237.

Kirsch, W., Pfister, R., \& Kunde, W. (2016). Spatial action-effect binding. Attention, Perception, \& Psychophysics, 78, 133-142.

Knill, D. C. (2005). Reaching for visual cues to depth: The brain combines depth cues differently for motor control and perception. Journal of Vision, 5(2), 2:103-115. https://doi.org/10.1167/5.2.2

Ladwig, S., Sutter, C., \& Müsseler, J. (2012). Crosstalk between proximal and distal action effects during tool use. Zeitschrift für Psychologie, $220,10-15$.

Ladwig, S., Sutter, C., \& Müsseler, J. (2013). Intra- and intermodal integration of discrepant visual and proprioceptive action effects. Experimental Brain Research, 231, 457-468.

Macaluso, E., Noppeney, U., Talsma, D., Vercillo, T., Hartcher-O’Brien, J., \& Adam, R. (2016). The curious incident of attention in multisensory integration: Bottom-up vs. top-down. Multisensory Research, 29, 557-583.

Milner, A. D., \& Goodale, M. A. (1995). The visual brain in action. Oxford, UK: Oxford University Press.

Milner, A. D., \& Goodale, M. A. (2008). Two visual systems re-viewed. Neuropsychologia, 46, 774-785. https://doi.org/10.1016/j. neuropsychologia.2007.10.005

Monsell, S. (2003). Task switching. Trends in Cognitive Sciences, 7, 134 140. https://doi.org/10.1016/S1364-6613(03)00028-7

Odegaard, B., Wozny, D. R., \& Shams, L. (2016). The effects of selective and divided attention on sensory precision and integration. Neuroscience Letters, 614, 24-28.

Paillard, J. (1991). Motor and representational framing of space. In J. Paillard (Ed.), Brain and space (pp. 163-182 ). Oxford, UK: Oxford University Press.

Rand, M. K., \& Heuer, H. (2013). Implicit and explicit representations of hand position in tool use. PLOS ONE, 8, e68471. 
Rand, M. K., \& Heuer, H. (2016). Effects of reliability and global context on explicit and implicit measures of sensed hand position in cursorcontrol tasks. Frontiers in Psychology, 6, 2056.

Rand, M. K., \& Heuer, H. (2017). Contrasting effects of adaptation to a visuomotor rotation on explicit and implicit measures of sensory coupling. Psychological Research. https://doi.org/10.1007/s00426017-0931-1.

Rossetti, Y., Desmurget, M., \& Prablanc, C. (1995). Vector coding of movement: Vision, proprioception, or both? Journal of Neurophysiology, 74, 457-463.

Sober, S. J., \& Sabes, P. N. (2005). Flexible strategies for sensory integration during motor planning. Nature Neuroscience, 8 , 490-497.

Song, J.-H., \& Nakayama, K. (2007). Automatic adjustment of visuomotor readiness. Journal of Vision, 7(5), 2:1-9. https://doi. org $/ 10.1167 / 7.5 .2$
Talsma, D., Doty, T. J., \& Woldorff, M. G. (2007). Selective attention and audiovisual integration: Is attending to both modalities a prerequisite for early integration? Cerebral Cortex, 17, 679-690. https://doi.org/ 10.1093/cercor/bhk016

Talsma, D., Senkowski, D., Soto-Faraco, S., \& Woldorff, M. G. (2010). The multifaceted interplay between attention and multisensory integration. Trends in Cognitive Sciences, 14, 400-410. https://doi.org/ 10.1016/j.tics.2010.06.008

van Beers, R. J., Sittig, A. C., \& Denier van der Gon, J. J. (1999). Integration of proprioceptive and visual position-information: An experimentally supported model. Journal of Neurophysiology, 81, $1355-1364$.

Zelaznik, H. N., Hawkins, B., \& Kisselburgh, L. (1983). Rapid visual feedback processing in single-aiming movements. Journal of Motor Behavior, 15, 217-236. 